\title{
Design of High-Power White LED Driver
}

\author{
ChaoJun Yan \\ College of Computer and Information technology, Three Gorges University, Yi Chang, China \\ aerolus@aliyun.com
}

Keywords: Driving circuit; LED; White light; XLT604; High-power; Illumination; Constant current; Dimmer

\begin{abstract}
The driving circuit for ten high-power white LED bulbs which are connected in series is designed. The core chip used is XLT604 and the peripheral circuits such as rectification circuit, dimming circuit are also designed. The modulation of LED is via the outer control voltage and therefore it belongs to a kind of linear dimming method. The parameters of key devices, such as the sampling resistance R6, the oscillation resistance $\mathrm{R} 4$, the inductance L1 and the fast restoring diode D5, are selected carefully according to relevant theoretical formula.
\end{abstract}

\section{Introduction}

In illumination domain, the application of LED productions is being paid widely attention $[1,2$, and 3$]$. As a new type of light source, it represents the developing trend in future. The fourth revolution in lighting industry is coming. Because of the lower working voltage of LED, the special driver circuit is needed to assist in providing energy. However, the driver technology of LED now is not well- matured and this is one of the important causes of influencing the popularization of LED illumination. The LED illumination possess the superiority of prime cost in some applied occasions because of its merits such as safety, long life, more types of color, infinitely flickering and handily control. In fact, it has been widely used in the domain of large power illumination.

In this paper, the driver circuit for a LED system with ten white LED bulbs is designed.

\section{Overvie w of LED drivers}

The properties of driver circuit for high- power white LED. Considering the energy conservation, LED sources are more efficient than ordinary sources. However, the LED sources cannot be supported directly by the power grid, by contraries, the power adapter is needed to supply the voltage and the current which the LED light need. This is called special power source for LED [4]. In our design example, this special power source is also called driving circuit which provide 3.0 4.3 voltage for a white LED light.

The main demands for drive circuit are as follows: 1) When many white LEDs connected in parallel, every LED need have the same current to ensure the uniform brightness; 2) The driver circuit needs lower power dissipation and small static current. The LED lights can be controlled by electronic switch which is belonged to the driver. When the switch is closed, the static current is usually less than $1 \mu \mathrm{A}$; 3) The maximum current of white LED can be set and the brightness of white LED can be adjusted; 4) There exists the complete protective circuits, such as the low-voltage locking, over-voltage protection, over-heating protection, open-circuit output and short-circuit protection; 5) Small size packaging, few peripheral components; 6) The interference to other circuits is less.

The classification of LED drivers. Because the property and power conversion efficiency of different types of drivers is different, in order to show the high efficiency of LED drivers, you must choose the most suitable LED driver for your design. The suitable driver will influence the stability, lifetime and degree of energy conservation of LED. 
The LED drivers can be roughly classified to two types. One is called constant current type (CC)[5]; the other is called constant voltage type (CV).

The $\mathrm{CC}$ driver is perfect but its price is more expensive. Although the $\mathrm{CC}$ driver can tolerate short circuit of load, but the completely open is strictly prohibited. The direct voltage of output varies in a certain range according to the load impedance even if the output current of CC driver is stable. The number of LED bulbs is limited in the application because the $\mathrm{CC}$ driver has the upper limits of the maximum working current and voltage.

The CV driver output a stable voltage; the output current varies according to the load impedance. Although the $\mathrm{CV}$ driver can tolerate the open circuit of load but the short circuit of load is strictly prohibited. The suitable current-limiting resistance is needed in order to homogenize the brightness of many LED bulbs.

Isolation and rectification. There exist two kinds of structures of drivers as to whether or not the drivers are isolated from the electric supply. The greatest danger of not-isolation driver is that all the LEDs will be damaged completely in case of the large current passing though the LEDs. Consequently, this driver put forward higher requirement for the insulation between the LEDs and the heat sink. If not, the body of light will be charged. The isolated-type driver has higher cost than no-isolated-type driver. It thus has higher security. The LEDs will not correspondingly be damaged if the driver breaks down. Inside the isolated-type driver, the common isolation processing between the LEDs and the heat sink can fulfill the standard of electrical isolation.

The most valuable method of power supply is directly from the public supply system and this is the problem which must be solved relating to the popularization of semiconductor illumination. The electric adapter is thus been induced. The adapter for the driver must be with high transform efficiency, smaller volume and lower cost. For the high-power application, the bridge rectifier is used.

In our design, the insolated driver and the bridge rectifying adapter are adopted.

The dimmer of LED drivers. The brightness of LED is proportional to the current. When you need higher brightness, you must raise the current passing through it. The usual dimming me thods comprise the pulse-width modulation (PWM)[6], analog methods and digital methods. The duty ratio of square wave is used to set the current of LED in PWM method. The PWM dimming is actually a switch technology, namely, the driver is turned on or turned off repeatedly according to a pulse signal with different widths.

\section{Design of a LED driver}

Because the voltages of each high-power LEDs are not consistent and the LED is much affected by the temperature of PN junction and the surroundings, so ten LEDs in our example [6] are connected in series and droved by $\mathrm{CC}$ driver to overcome these shortcomings. The $\mathrm{CC}$ driver can avoid the current fluctuation caused by the changes of forward voltage, so the LEDs have the same brightness.

The parameters of LEDs[7,8] are as follows:1) white; 2)working voltage,3.0-3.2V; 3) rated current,350mA, corresponding power, $1 \mathrm{~W}$; 4) emitting angle, 120 degree; 5) color temperature, 5500-7000K; 6) brightness, 70-80 Lumina.

XLT604 Integrated chip. In our design, the chip XLT604 which is built with BICMOS technology is chosen as the LED controller. The power LEDs can be driven efficiently in the range of input voltage 8-450V. The advantages of this chip are its broad range of constant current, the high efficiency and easy controlled. It can drive the outer MOS-FET with the fixed $300 \mathrm{KHz}$ frequency and this frequency can set by the outer resistance. The power LEDs are driven by $\mathrm{CC}$ driver and the value of constant current whose variation range is from some milliampere to one ampere can be set through the outer sample resistance. The brightness of LEDs driven by XLT604 can be lineally modulated by outer control voltage or by the method of outer low-frequency PWM[9,10]. 
The properties of XLT604 are as follows: 1) the source of chip is $6.1 \mathrm{~V} ; 2$ ) greater efficiency, over 90\%; 3 ) the range of input voltage is from $7 \mathrm{~V}$ to $450 \mathrm{~V}$ or $\mathrm{AC}$ input; 4) $\mathrm{CC}$ type, the range of CC is from $5 \mathrm{~mA}$ to $1 \mathrm{~A}$; 5) the maximum LEDs driven is 100 ; 6) outer linearly modulation or PWM modulation.

The insider structure block of XLT604 is shown in figure one.

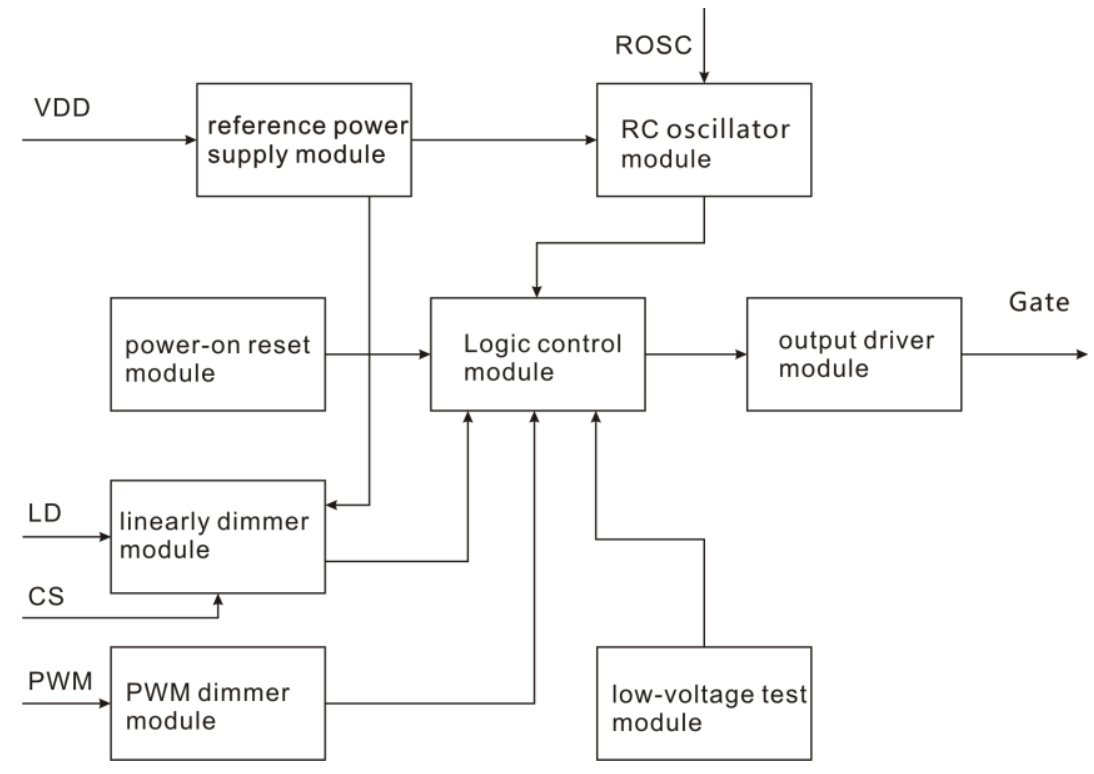

Fig. 1 The insider structure block of XLT604

The calculating of parameters of related devices. The circuit diagram of driver circuit is shown in figure two. The parameters of main components in the circuit diagram are calculated as follows.

The sampling resistance, $\mathrm{R} 6$

The maximum output current of LEDs used is $350 \mathrm{~mA}$,. As we known, this current is equal to 0.25/R6; therefore, the value of R6 is about $0.71 \mathrm{ohm}$.

The oscillation resistance, $\mathrm{R} 4$

There exist two methods of modulation of XLT604: one is that the square wave acted as PWM to set the current of LED is accessed through the pin 7 of XLT604; another is call linear modulation, nameless, the current of LEDs is changed according to the value of oscillation resistance R4. In our design, the second method is used. The inner oscillation frequency is determined by the outer resistance R4. The design formula is: ${ }^{\text {freq }}=22000 /(R 4+22)$, where the parameters such as 22000 and 22 are intrinsic RC parameters. The typical value of $f r e q$ is $20-150 \mathrm{KHz}$. This value is relevant to the inductance $\mathrm{L} 1$ which will be discussed later. In general, the $\mathrm{L} 1$ is little and the freq is much. In our design, freq $=33 \mathrm{KHz}$, so, $R 4=680 \mathrm{~K}$.

The inductance, $\mathrm{L} 1$

Suppose the effective value of input voltage is $220 \mathrm{~V}$, the output current is $350 \mathrm{~mA}$, freq $=33 \mathrm{kHz}$, the forward-voltage drop of ten LEDs is $V_{\text {out }}=30 \mathrm{~V}$, therefore, the voltage after rectification is $V_{\text {in }}=220 \times 1.414=310 \mathrm{~V}$.The duty ratio of switch $D=V_{\text {out }} / V_{\text {in }}=30 / 310=0.097$,so.

The fast restoring diode, D5

The fast restoring diode is the main source of high-frequency noise. It is important to select a good fast restoring diode. We select this diode mainly according to two parameters: the maximum average current and the fast restoring time. In general, we choose the diode FR307 with the parameters such as: the maximum 
average current $2 \mathrm{~A}$ and the restoring time $150 \mathrm{~ns}$. In order to reduce the noise of power source, a $100 \mathrm{pF}$ capacitance $\mathrm{C} 8$ is connected in parallel.

The operating principle of circuit. The alternating current (A.C.) of $220 \mathrm{~V}$ is changed to about $310 \mathrm{~V}$ via the filtering and rectifying (D1, D2, D3, D4, C3) circuit and the ripple wave is little. This voltage is then divided by a voltage-splitter resistance (R1), finally, by means of voltage regulator diode $\mathrm{D} 7,6.2 \mathrm{~V}$ voltage whose rising speed is increased via C4 is provided to XLT604 as power supply and the $6.2 \mathrm{~V}$ voltage is stabilized via C2.Organization of the Text

When the GATE pin of XLT604 is high level, the MOSFET Q1IRF840 is opened. The current flows through the serials of LEDs, The inductance L1, Q1, sampling resistance R6 and finally to ground. Due to the property of inductance, in the period of high level of GATE pin, the current is gradually increased. When the voltage of R6 reaches $0.25 \mathrm{~V}$, the voltage of GATE pin turn to low level and Q1 is turned off. The energy stored in L1 is released through the fast restoring diode D5 to maintain the current of LEDs. In this process, the current through LEDs is also gradually descended untilL1 begin to be charged when the next high level appears in the GATE pin. The constant current source needed by LEDs is so provided via these repeatedly charging and discharging. The frequency of current (charging and discharging) is determined by the oscillation source inside the chip, but this frequency can be adjusted via the outside resistance R4.If the current is little, for example, under 100mA,the resistance R4 should be decreased in order to increase the oscillation frequency.



Fig. 2 The circuit diagram of LED driver

\section{Conclusions}

In this paper, a driving circuit for ten high-power white LED bulbs which are connected in series is designed. The core chip is XLT604 and the peripheral circuits such as rectification circuit, dimming circuit are also included. The modulation of LEDs is via the outer control voltage and therefore it belongs to a kind of linear dimming method. The parameters of key devices, such as the sampling resistance R6, the oscillation resistance $\mathrm{R} 4$, the inductance L1 and the fast restoring diode D5, are selected carefully according to relevant theoretical formula. Proven by the real objects built according our design, this CC driving circuit can supply stable and reliable power for LED light. 


\section{References}

[1] YUTANG Lei and LI Hui, "Lighting source in the future-White light LED," Optics \& Optoelectronic Technology, vol.1(5),pp. 33-34.

[2] Z. ZhiMing,Z. JiHai and J. AiHua,Design and application of LED drive circuit. BeiJing:The People's Posts and Telecommunications Press,2006.

[3] Z. ZhiMing,Z. JiHai and J. AiHua, the lighting technique and application of LED. BeiJing:Electronic Industry Press, 2009.

[4] Z. ZhiMing,Z. JiHai and J. AiHua,The design examples of drive circuits. BeiJing:Electronic Industry Press, 2007.

[5] Mehm Nalbant, "High-Efficiency Current Drive for High-Brightness LED," Electronic Engineering \& Product World,2005, (9),pp.101-103.

[6] Z. ZhiMing,Z. JiHai and J. AiHua, The application circuits of Switching Power Supply. BeiJing: China Electric Power Press, 2006.

[7] Li Zhihang and Gao Tiecheng, "LED Road Lighting Source with the Heat and Light Application," Electrical Engineering,2008,(7),pp.95-98

[8] Nagel A,Doncker D,R.W. Systematic design of EMI-filters for power converters. Industry Applications Conference,2000,2523-2525

[9] Lo,Y.K.\&S.C.Yen, A High-Efficiency AC-to-DC Adaptor with a low standby Power Consumption[J] , Industrial Electronics,IEEE Transactions on,55[2],Feb.2008,963-965

[10] Abdel-Salam M,Ahmed N.A, Elhamd I S. Varistor as a surge protection device dor electronic equipments. Industrial Technology,2004,668-694 